\title{
Cell Assemblies in the Cortico-Hippocampal-Reuniens Network during Slow Oscillations
}

\author{
${ }^{-}$David Angulo-Garcia, ${ }^{1 *}{ }^{\circledR}$ Maëva Ferraris, ${ }^{2 \star}$ Antoine Ghestem, ${ }^{2}{ }^{\circledR}$ Lauriane Nallet-Khosrofian, ${ }^{2}$ \\ Christophe Bernard, ${ }^{2}$ and ${ }^{-}$Pascale P. Quilichini ${ }^{2}$ \\ ${ }^{1}$ Grupo de Modelado Computacional, Instituto de Matematicas Aplicadas, Universidad de Cartagena, Cartagena de Indias, 130001, Colombia, and \\ ${ }^{2}$ Aix-Marseille Univ, INSERM, INS, Inst Neurosci Syst, 13005, Marseille, France
}

The nucleus reuniens (NR) is an important anatomic and functional relay between the medial prefrontal cortex (mPFC) and the hippocampus (HPC). Whether the NR controls neuronal assemblies, a hallmark of information exchange between the HPC and mPFC for memory transfer/consolidation, is not known. Using simultaneous local field potential and unit recordings in NR, HPC, and MPFC in male rats during slow oscillations under anesthesia, we identified a reliable sequential activation of NR neurons at the beginning of UP states, which preceded mPFC ones. NR sequences were spatially organized, from dorsal to ventral NR. Chemical inactivation of the NR disrupted mPFC sequences at the onset of UP states as well as HPC sequences present during sharp-wave ripples. We conclude that the NR contributes to the coordination and stabilization of mPFC and HPC neuronal sequences during slow oscillations, possibly via the early activation of its own sequences.

Key words: cell assemblies; hippocampus; network; nucleus reuniens; medial prefrontal cortex; slow oscillations

\section{Significance Statement}

Neuronal assemblies are believed to be instrumental to code/encode/store information. They can be recorded in different brain regions, suggesting that widely distributed networks of networks are involved in such information processing. The medial prefrontal cortex, the hippocampus, and the thalamic nucleus reuniens constitute a typical example of a complex network involved in memory consolidation. In this study, we show that spatially organized cells assemblies are recruited in the nucleus reuniens at the UP state onset during slow oscillations. Nucleus reuniens activity appears to be necessary to the stability of medial prefrontal cortex and hippocampal cell assembly formation during slow oscillations. This result further highlights the role of the nucleus reuniens as a functional hub for exchanging and processing memories.

\section{Introduction}

Information exchange between the hippocampus (HPC) and the medial prefrontal cortex (mPFC) is essential for different memory processes, including consolidation (Siapas and Wilson, 1998; Frankland and Bontempi, 2005; Preston and Eichenbaum, 2013; Maingret et al., 2016; Eichenbaum, 2017; Kitamura et al., 2017; Latchoumane et al., 2017; Ferraris et al., 2018). The representation of information is supported by the recruitment of neuronal cell assemblies, which refer to a group of neurons that are

\footnotetext{
Received Mar. 9, 2020; revised July 22, 2020; accepted Sep. 1, 2020.

Author contributions: D.A.-G., M.F., and P.P.Q. analyzed data; D.A.-G. and P.P.Q. wrote the first draft of the paper; D.A.-G., M.F., C.B., and P.P.Q. edited the paper; D.A.-G. and P.P.Q. wrote the paper; M.F. and P.P.Q. designed research; M.F., A.G., L.N.-K., and P.P.Q. performed research'

${ }^{*}$ D.A.-G. and M.F. contributed equally to this work.

The authors declare no competing financial interests.

This work was supported by Fondation Recherche Médicale Grant FDT201805005246 to M.F. D.A.-G. was supported by A*MIDEX Grant ANR-11-IDEX-0001-02 and Vicerrectoria de Investigaciones-Universidad de Cartagena Project 038-2019. We thank Wesley Clawson for proofreading the manuscript.

Correspondence should be addressed to Pascale P. Quilichini at pascale.quilichini@univ-amu.fr.

https://doi.org/10.1523/JNEUROSCI.0571-20.2020

Copyright $\odot 2020$ the authors
}

coactivated repeatedly for a given brain operation and thus represent a distinct entity embedded within neuronal networks (Hebb, 1949; Buzsáki, 2010; Buzsaki, 2019). In both HPC and mPFC, cell assemblies fire in a fine time-resolved manner (Skaggs and McNaughton, 1996; Nadasdy et al., 1999; Lee and Wilson, 2002; Euston et al., 2007; Luczak et al., 2007; Pastalkova et al., 2008; Peyrache et al., 2009; Battaglia et al., 2011). These neuronal assemblies display a precise temporal sequential activation, which reflects the encoded information (Nádasdy, 2000; Foster and Wilson, 2006; Ji and Wilson, 2007; Davidson et al., 2009; Marre et al., 2009; Pfeiffer, 2020). Such activity is prevalent during sleep (Pavlides and Winson, 1989; Wilson and McNaughton, 1994; Nadasdy et al., 1999; Atherton et al., 2015), particularly during NREM sleep, and is organized in space and time by a set of oscillations, such as hippocampal sharp-wave ripples (SPW-Rs), cortical slow oscillations (SOs), and spindles (Sirota et al., 2003; Sirota and Buzsáki, 2005; Staresina et al., 2015; Maingret et al., 2016). How these cell assemblies are finely coordinated between the HPC and the mPFC to support memory consolidation is not well understood. The thalamic nucleus reuniens (NR), which bidirectionally connects the HPC and the 
mPFC (Herkenham, 1978; Van der Werf et al., 2002; Vertes, 2006; Varela et al., 2014), plays a key role in memory consolidation (Loureiro et al., 2012; Cassel et al., 2013; Pereira de Vasconcelos and Cassel, 2015; Dolleman-van der Weel et al., 2019; Klein et al., 2019). In particular, the NR synchronizes $\gamma$ bursts, which can open temporal windows for information exchange (Buzsáki and Draguhn, 2004; but see Buzsáki and Schomburg, 2015), between the HPC and the mPFC during SOs (Ferraris et al., 2018). The NR is therefore ideally poised to orchestrate the dynamics of cell assemblies in both regions.

We have recorded and manipulated the NR-mPFC-HPC circuit during SOs under anesthesia, which resembles sleep patterns and provides long periods for analysis. We find that cell assemblies are recruited in the NR at the onset of the UP state of SOs and that NR activity is needed for the stability of HPC and mPFC cell assemblies.

\section{Materials and Methods}

Experimental model and subject details. All experiments were performed in accordance with experimental guidelines approved by Aix-Marseille University Animal Care and Use Committee under the authorization \#01451-02. All rats were group housed to avoid social isolation-induced stress (Manouze et al., 2019). A total of 18 rats were used in this study. Part of these data (14 Wistar Han rat data) was used in a previously published study (Ferraris et al., 2018), and 4 Wistar Han rats are original data. They include local field potentials (LFPs) and singleunit recordings made in the $\mathrm{MPFC}, \mathrm{HPC}$, and NR of anesthetized rats.

Animal surgery. Wistar Han IGS male rats (250-400 g; Charles River; RRID:RGD_2308816) were anesthetized with urethane (1.5 g/kg, i.p., catalog \#U2500, Sigma Millipore) and ketamine/xylazine (20 and $2 \mathrm{mg} / \mathrm{kg}$, i. m., Renaudin Cip: 3400957854195 and Centravet, catalog \#ROM001, respectively), additional doses of ketamine/xylazine ( 2 and $0.2 \mathrm{mg} / \mathrm{kg}$ ) being supplemented during the electrophysiological recordings. The heart rate, breathing rate, pulse distension, and the arterial oxygen saturation were also monitored with an oximeter (MouseOx, Starr Life Science) during the entire duration of the experiment to ensure the stability of the anesthesia and monitor the vital constants. The head was secured in a stereotaxic frame (Kopf \#962, Phymep), and the skull was exposed and cleaned. Two miniature stainless-steel screws, driven into the skull above the cerebellum, served as ground and reference electrodes. Up to three craniotomies were performed to target, from bregma: the prelimbic area of the $\mathrm{mPFC}$ at $3 \mathrm{~mm} \mathrm{AP}$ and $0.8 \mathrm{~mm} \mathrm{ML}$; the CA1 field of the intermediate $\mathrm{HPC}$ at $-5.6 \mathrm{~mm} \mathrm{AP}$ and $4.3 \mathrm{~mm} \mathrm{ML}$; and the NR at $-1.8 \mathrm{~mm} \mathrm{AP}$ and $-2 \mathrm{~mm}$ ML. Silicon probes (NeuroNexus) were used to record from these structures: a A1x32-Edge-5 mm-20-177-H32-15 probe placed at $[-2.5-3.1] \mathrm{mm}$ from brain surface to reach mPFC layer 5; a A1x32Edge-10 mm-20-177-H32-50 32-site probes placed at $-7.2 \mathrm{~mm}$ from brain surface to reach the NR; and a HPC A1x32-6 mm-50-177-H32-15 probe placed at $[-2.8-3.0] \mathrm{mm}$ perpendicularly to the CA1 field from stratum oriens to stratum lacunosum moleculare in the HPC. All the probes were lowered inside the brain with a motorized manipulator (IVM single, Scientifica).

For NR inactivation experiments ( $n=5$ rats), a local injection of a fluorophore-conjugated muscimol (BODIPY-MSCI TMR-X Conjugate, catalog \#M23400, Invitrogen) was performed in the NR, and data from the $\mathrm{mPFC}$ and the HPC (CA1) were simultaneously acquired. The injection needle (33 gauge, catalog \#87930, catalog \#7803-05, Hamilton) was inserted in the NR (using the same depth coordinates as the probes and mounted on the same micromanipulator) and $0.70 \mathrm{nmol}$ of muscimol in $0.3 \mu \mathrm{l}$ of PBS (Ferraris et al., 2018) was delivered over $60 \mathrm{~s}$ through a micropump (UltraMicroPump UMP3-1, WPI). The needle was left in place for 3 additional minutes to allow for adequate diffusion of the drug, then carefully removed.

At the end of the recording, the animals were injected with a lethal dose of pentobarbital $\mathrm{Na}(150 \mathrm{mg} / \mathrm{kg}$, i.p.) and perfused intracardially with $4 \%$ PFA solution in $\mathrm{PB}(0.12 \mathrm{M})$. The position of the electrodes (DiIC18(3), catalog \#46804A, InterChim) was applied on the back of the probe before insertion and was confirmed histologically on Nissl-stained $60 \mu \mathrm{m}$ sections (NeuroTrace 500/5225 Green Fluorescent Nissl Stain, catalog \#N21480, Invitrogen). Only experiments with appropriate position of the probe were used for analysis (see Fig. 3A).

Data collection and initial analysis. Extracellular signal recorded from the silicon probes was amplified $(1000 \times)$, bandpass filtered $(1 \mathrm{~Hz}$ to $5 \mathrm{kHz}$ ), and acquired continuously at $32 \mathrm{kHz}$ (64-channel DigitalLynx; NeuraLynx) at 16-bit resolution. Raw data were preprocessed using a NEUROSUITE (Hazan et al., 2006; http://neurosuite.sourceforge.net/; RRID:SCR_008020). After recording, the signals were downsampled to $1250 \mathrm{~Hz}$ for the LFP. Spike sorting was performed automatically using KLUSTAKWIK (Harris et al., 2000) (http://klustakwik.sourceforge.net/; RRID:SCR_008020; RRID:SCR_014480), followed by manual adjustment of the clusters, with the help of autocorrelogram, cross-correlogram, and spike waveform similarity matrix (KLUSTERS software, RRID:SCR_008020) (Hazan et al., 2006). After spike sorting, the spike features of units were plotted as a function of time, and the units with signs of substantial drift over the period of recording were discarded. Moreover, only units with clear refractory periods and well-defined cluster were included in the analyses (Harris et al., 2000). Recording sessions were divided into brain states of theta and SO periods. The epochs of stable SO periods were visually selected respectively from the ratios of the whitened power in the SO band $([0.52] \mathrm{Hz})$ and the power of the neighboring band $\left(\left[\begin{array}{ll}20 & 30\end{array}\right] \mathrm{Hz}\right)$ of $\mathrm{mPFC}$ or NR LFP and assisted by visual inspection of the raw traces (Quilichini et al., 2010).

Neurons were assigned as "NR neurons" by determining the approximate location of their somata relative to the recording sites, the known distances between the recording sites, the histologic reconstruction of the recording electrode tracks, and subsequent estimation of the recording sites. All the neurons recorded from sites located near the close contour of the NR were discarded. Neurons located at a minimal distance of $200 \mu \mathrm{m}$ of NR border and located within contours of the ventro-median, submedian or antero-median thalamic nuclei were classified as "other thalamic neurons" and used in the analysis (Ferraris et al., 2018).

Data postprocessing. All the analysis was performed using customwritten MATLAB (RRID:SCR_001622; RRID:SCR_008020) scripts. From the spike times, the instantaneous firing rates of each cell were calculated by counting the number of spikes inside a window of $50 \mathrm{~ms}$, in overlapping intervals of $10 \mathrm{~ms}$. The population firing rate was estimated by averaging the single-cell firing rates at each $10 \mathrm{~ms}$ interval. Both the population rate and the single-cell firing rate were smoothed with a Gaussian kernel of $5 \mathrm{~ms}$ width. Peaks of population activity (AP) were identified as the points where the population rate amplitude was larger than the average rate plus $1 \mathrm{SD}$. A separation between two consecutive peaks of at least $600 \mathrm{~ms}$ was imposed on the peak detection algorithm to avoid multiple peaks of activity within one UP state. For visualization purposes, all firing rate's heat maps were normalized with the peak firing rate for each cell to guarantee a variation between [0:1].

Phase analysis and sequence identification. LFP signals (from $\mathrm{mPFC}$ or NR) during SO phase were bandpass filtered between 0.5 and $2 \mathrm{~Hz}$ with a second-order Butterworth filter to extract only the UP/DOWN transitions. The time evolution of the phase during the UP/DOWN cycle was extracted performing the Hilbert transform of the filtered LFP (from the mPFC or the NR) and was visually checked. The [0.5 2] Hz LFP oscillation is most likely volume-conducted from the cortex in the NR (for review, see Buzsáki et al., 2012; Herreras, 2016), and this was checked by computing the current source density analysis as the second spatial derivative of the NR LFPs and smoothed spatially with a triangular kernel (Quilichini et al., 2010; Ferraris et al., 2018), which resulted in a flat signal on all the channels (data not shown).

Similar results regarding the detection of UP/DOWN transitions were obtained by constructing the phase as a linear interpolation between peaks and troughs of the filtered LFP signals (Belluscio et al., 2012). We also obtained similar results by extracting UP states from the mPFC multiunit activity. UP states were identified as periods of neuronal firing between periods of at least $100 \mathrm{~ms}$ of global silence (without spike). The time of the first spike marking a transition between the silence period and the active one is defined as the onset of the UP state. UP states offset corresponds to the time of the last spike marking a 
A a

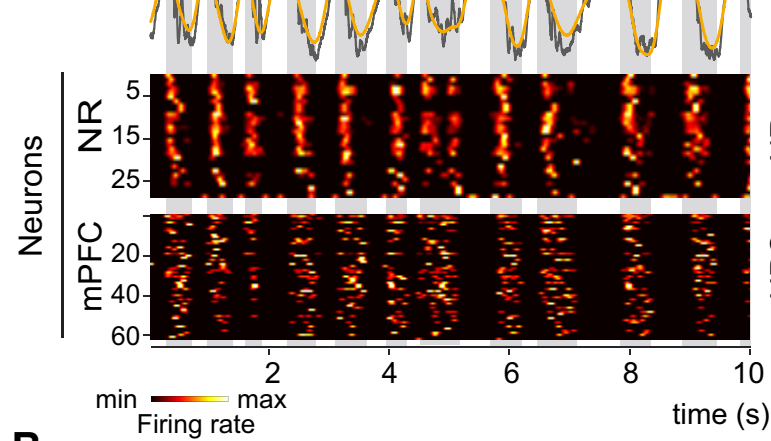

B

a

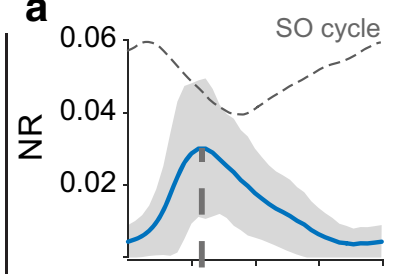

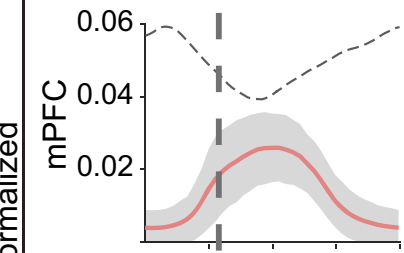
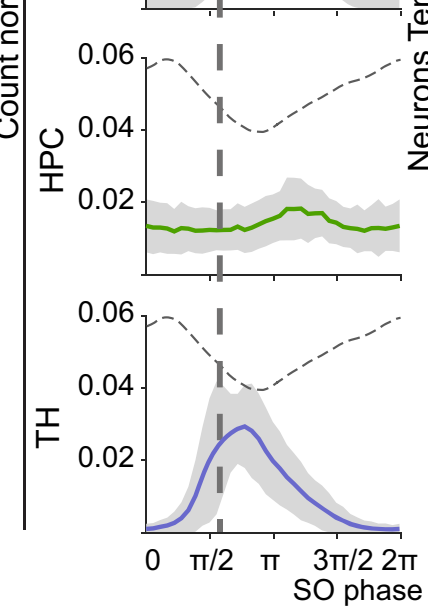

b NR

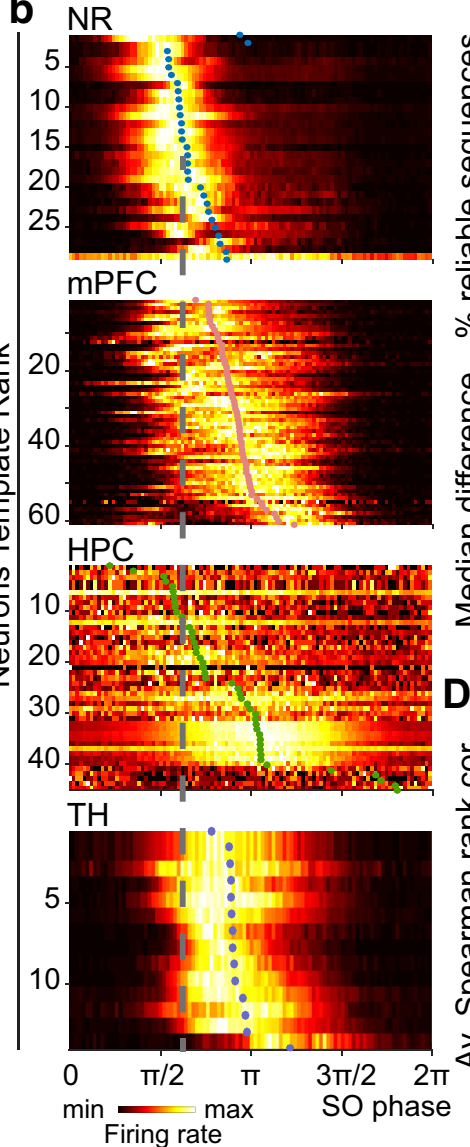

D b

NR LFP + SO filtered LFP

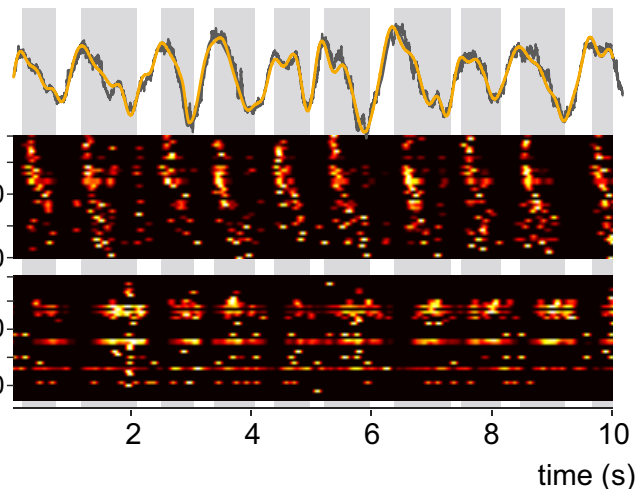

C
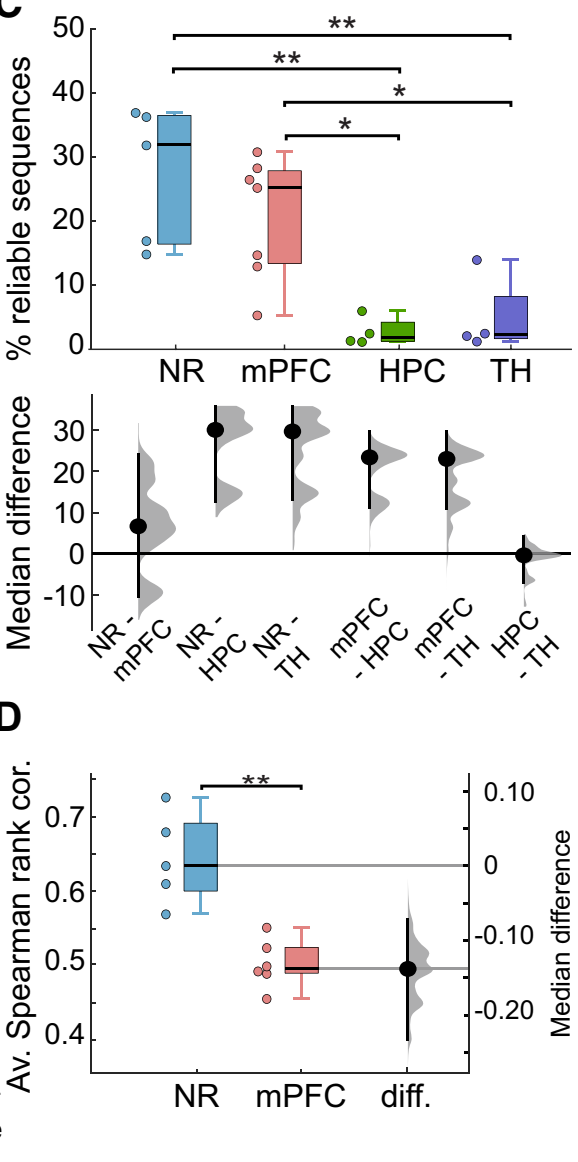

Figure 1. Sequential dynamics of cell assemblies. $\boldsymbol{A}$, Top, LFPs from (Aa) mPFC and (Ab) NR (mainly volume-conducted from the cortex) in gray, with superimposed [0.5 2] Hz filtered LFPs (yellow), where the UP states are marked by the gray boxes, from two typical recordings. Bottom, The corresponding template heat maps of normalized NR unit activities with (Aa) mPFC or (Ab) HPC normalized unit activities show the repetition of neuronal assemblies, particularly in the NR and the MPFC. The identification number of the recorded neurons is indicated on the left axis of the heat maps. $B \boldsymbol{a}$, Phase distribution of the NR, mPFC, HPC, and TH population firing (grouped data, all recordings) with reference to the SO phase (indicated by the dashed curve). Dashed line indicates the most preferred phase of NR neurons, showing that their firing precedes other cells located in the mPFC, HPC, and TH at the onset of the UP state. $B \boldsymbol{B}$, Heat maps of normalized distribution of neurons preferred SO phase for sample representative firing patterns of NR (same neurons as in $\boldsymbol{A a}$ ), mPFC (same as in $\boldsymbol{A a}$ ), HPC (same as in $\boldsymbol{A} \boldsymbol{b}$ ), and TH (representative example). The activation order for each neuron is calculated as the average preferred phase. Heat maps are ordered according to the increasing value of average preferred phase (colored circles), defining a template order. The sequential activation is clearly apparent in NR and MPFC neurons, but neither in the HPC nor in the TH (the phase distribution extends over at least $\pi / 2$ for each neuron). C, Top, The largest percentage of reliable sequences is found in the NR and mPFC compared with the HPC and the TH. Bottom, The median differences for 6 comparisons are shown in a Cumming estimation plot. Each median difference is plotted on the lower axis as a bootstrap sampling distribution. Dots represent median differences. Ends of the vertical error bars represent $95 \%$ Cls. $\left({ }^{*} p<0.05,{ }^{* *} p<0.01\right) \boldsymbol{D}$, The average Spearman correlation shows that sequences in NR are more reliable than those of mPFC. The estimation plot on the right side shows the median difference (dots) and the $95 \%$ Cl (diff., median difference).

transition to a silence period. UP states $<200 \mathrm{~ms}$ were excluded (Luczak et al., 2007). We used circular statistics (Berens, 2009; Ferraris et al., 2018) to compute the mean phase at which each neuron fires ("preferred phase") and to build their firing-phase histograms (see Fig. 1Ba). For visualization purposes, the resulting histograms depicted in the heat maps were normalized with the peak value of the distribution. For each cell, we then calculated a resultant vector characterized by an angle describing the average preferred phase and a magnitude with values between [0 1], quantifying the coherence of the phases. The template order was obtained by organizing the average preferred phase in increasing order $\left[\begin{array}{lll}0 & 2 & \pi\end{array}\right]$. To prove that sequential ordering was not because of purely random fluctuations, we also calculated the preferred phase using half of the 
recording and compared with the preferred phase using the second half of the recording. Moreover, to test the possibility that sequences were a consequence of differences in firing rates between neurons, we normalized the firing rate of all recorded units in such a way that all neurons had an average $2 \mathrm{~Hz}$ firing rate by means of random spike removal. After spike removal, the average phases were recalculated and compared with the average phases obtained with the unnormalized spike train.

Sequence reliability, participation index, and sequence delay. We extracted the UP state duration as described by Ferraris et al. (2018). At each UP state, the local activation order was calculated by measuring the time lag between the first activation of each neuron relative to the AP in a $\pm 200 \mathrm{~ms}$ window. Ordering the latencies in increasing order resulted in the local activation order. The reliability of a given sequence within a population peak was quantified as the Spearman rank correlation of the template order and the local activation order in that particular UP state. Sequences were considered reliable above $99 \%$ significance level. For each reliable sequence, a robust fit between the local activation order and the activation latency was computed. Nonparticipating neurons in a given sequence were identified as neurons that meet one of the following criteria: (1) did not fire within the $\pm 200 \mathrm{~ms}$ window or (2) neurons appearing as outliers in the robust fit (those whose residual value were larger than twice the SD of the residuals in the robust fit). The participation index was then calculated as the mean fraction of neurons that participated in the sequences of a recording session. A second linear fit between the local activation latency and the site of the linear probe closest to the neuron gave a measure of the directionality of the sequence activation. A slope of the fit different from 0 implies an activation toward a given direction. Since this slope has units of time/electrode site, we multiplied the resulting value by 32 (the number of sites in the electrode) to account for the time required for a sequence (sequence delay) to travel along the electrode.

Activity peak triggered histogram (APTH). Once the APs have been identified according to the algorithm described in Data postprocessing, we store the values of activation time lag of the first spike of each neuron relative to the AP at each UP state in a time window of $\pm 200 \mathrm{~ms}$ centered at the AP. This allows us to compute the so-called activity peak triggered histogram for each cell. With the APTH, it is possible to assess the statistics of the firing latencies in the neighborhood of the UP state. Once the APTH was obtained, one can calculate the variability of the activation lags as a measure of the firing extent around the peak reported in Figure 4C. Variability of the APTH can be calculated in analogy to the variance of a probability distribution function where $\tau$ is the mean value of the APTH (Fig. $4 C$, inset).

SPW-Rs detection and analysis. The procedure of SPW-Rs detection in the HPC stratum pyramidale LFP was based on those described previously (Isomura et al., 2006; Ferraris et al., 2018). Briefly, the LFP was digitally bandpass filtered [ 80250$] \mathrm{Hz}$, and the power (root-mean-square) of the filtered signal was calculated. The mean and SD of the power signal were calculated to determine the detection threshold. Oscillatory epochs with a power of $\geq 5$ SDs above the mean were detected. The beginning and the end of oscillatory epochs were marked at points where the power fell $0.5 \mathrm{SD}$. Once the SPW-Rs were detected, the SPW-R duration was calculated as the average between the start and the end of it. SPW-Rs are known to replay forward and backward sequences. With this in mind, we checked for different sequential activations in SPW-Rs by extracting the local firing order of neurons at each SPW-R (using the first spike as the activation latency for each neuron) and calculating a similarity matrix (cross-correlation) among all the obtained orders. The existence of well-defined clusters in the cross-correlation matrix could signal the existence of two or more different templates.

Therefore, we clustered the similarity matrices of the local orderings for each recording using a $k$-means algorithm with $k=1,2,3,4,5$. The optimal clustering is the partition in $k$ clusters that maximizes the modularity measure (for details, see Newman, 2010). For all the HPC recordings, modularity did not significantly change between $k=1$ and $k=2$ and definitely decreased for $k>2$. This means that there is no convincing evidence of the existence of different templates, and we assumed the existence of a unique template. With this, the APTH for each cell was computed, taking each SPW-R half-time as the activity peak. To test whether a cell robustly participates in the SPW-Rs, we compared the APTH against a uniform distribution with identical mean and SD. Cells whose APTH were different from the flat distribution above a 95\% level were considered as robustly participating in the SPW-Rs. Cell participation ratio was obtained dividing the number of robustly participating neurons by the total number of recorded neurons for that session. Organizing the average time lag for robustly participating neurons in increasing order defined the template order for the ripple. For each SPW-R, the reliability of activation respect to the template was calculated via the Spearman rank correlation between the activation order of that SPW-R and the template order. To calculate this correlation, only the neurons that belong to the template order were considered. A correlation $>95 \%$ CI was considered reliable.

Statistics. All results reported are based on a significance threshold $\alpha$ $=0.05$ (otherwise stated), and all groups included enough samples to enable rejection of the null hypothesis at that level. We used two-sample Kolmogorov-Smirnov test to assess differences between distributions, and Student's $t$ test to evaluate differences in the mean of distributions unless otherwise stated. In parallel, results from small samples data $(n<10)$ were evaluated using estimation statistics providing the effect size and the $95 \%$ CI of the median difference between two groups. Raw data were processed at https://www.estimationstats.com, directly giving both results and graphs (Calin-Jageman and Cumming, 2019). The median difference between two groups is shown with a Gardner-Altman estimation plot. Raw data are plotted on the left axis. The median difference is plotted on a floating axis on the right as a bootstrap sampling distribution (see Figs. $1 C, D, 3 D, 4 C, E, 5 B$ ). Five thousand bootstrap samples were taken; the $\mathrm{CI}$ is bias-corrected and accelerated. The mean difference is depicted as a dot; the $95 \%$ CI is indicated by the ends of the vertical error bar. If $95 \% \mathrm{CI}$ includes 0 , differences are considered as nonsignificant. The $p$ value(s) reported are the likelihood(s) of observing the effect size(s), if the null hypothesis of zero difference is true. Correlation tests involving ranked variables (neuron indices and electrode sites) were performed via a Spearman rank correlation. We tested significant differences between percentages with a two-proportion $Z$ test.

\section{Results}

\section{Cell assemblies are recruited in NR at UP state onset}

We first assessed the behavior of NR ( $n=166$ cells, $n=5$ rats), $\operatorname{mPFC}(n=496, n=7)$, and HPC $(n=163, n=4)$ neurons during SOs in anesthetized rats. SOs during anesthesia share similar features as NREM sleep (Hutt, 2011; Ferraris et al., 2018) and offer long-duration stable recordings necessary to identify statistically significant sequences. Neighboring thalamic neurons (anteromedian and ventro-median nuclei) were also recorded as a control group (“TH," 89 neurons, $n=4$ ).

During SOs, most of the neurons fired at the onset of the UP states, mainly in mPFC and NR (Fig. 1A) (Ferraris et al., 2018). We found a robust recruitment of neuronal activity at the UP state onset in NR (median percentage of recruited neurons per UP state $70.4 \%, \mathrm{~min} / \mathrm{max}: 61.1 \% / 85.9 \%$ ) and $\mathrm{mPFC}$ (median: $62.9 \%$, $\mathrm{min} / \max : 42.1 \% / 70 \%)$. Given the number of recorded neurons per session, $98 \%$ of the total number of UP states recorded in $\mathrm{mPFC}$ and $\mathrm{NR}$ recruit $>10$ neurons. In comparison, HPC neurons showed less participation during UP state (median: $33.8 \%, \mathrm{~min} / \max$ : $29.3 \% / 46.4 \%)$. To identify any ordering in this firing activity, we ranked each neuron according to its mean preferred SO phase (Fig. $1 \mathrm{Ba}$ ). In contrast, HPC neurons displayed better sequential activation during sharp-wave ripples (SPW-Rs), which tend to occur during the UP state (Sirota et al., 2003; Battaglia et al., 2004; Isomura et al., 2006; Buzsáki, 2015; Maingret et al., 2016; Khodagholy et al., 2017) (median: 45\%, $\min / \max : 35 \% / 64 \%)$.

Neurons were organized in increasing order of average preferred phase, defining a template order (Fig. $1 B b$ ). Sequences in 
NR always preceded mPFC ones (Fig. $1 B$ ). As a negative control, we analyzed the activity of neighboring TH neurons. Although $\mathrm{TH}$ neurons displayed a strong entrainment by SOs (Ferraris et al., 2018), there was poor sequential activity (Fig. 1B). The sequential activity at UP state onset therefore appears to be specific of $\mathrm{NR}$ and $\mathrm{mPFC}$ neurons in the areas investigated here. As a further control, we analyzed the activity of NR neurons during epochs dominated by theta $([46] \mathrm{Hz})$ oscillations. We did not find stable sequential neuronal assemblies during theta (data not shown). Together, these results show that, at the beginning of UP states, sequential cell assembly formation occurs first in the $\mathrm{NR}$, then in the mPFC, and marginally in the HPC and neighboring thalamic nuclei.

We then quantified the reliability of these sequences. To do so, we computed at each UP state the activation latency of each neuron relative to the population peak activity (see Materials and Methods). The local activation order of the cell assembly was determined from the ascending sorting of the activation latencies. We then calculated the Spearman rank correlation between the local activation order and the template order. A rank correlation $r=1$ indicates that the sequential activation follows exactly the template order determined previously by the average phase preferences. For NR neurons, the template order was significantly expressed in $32 \%$ of the total number of UP states (min/ $\max =15 \% / 37 \%$, Spearman test, $p<0.01$ ), while $\mathrm{mPFC}$ reliable sequences were found in $25 \%$ of the UP states $(\mathrm{min} / \mathrm{max}=5 \%$ / $31 \%$; Fig. 1C). In contrast, reliable HPC sequences were only detected in $2 \%$ of the UP states $(\mathrm{min} / \mathrm{max}=1 \% / 6 \%)$ and in $2 \%$ for sequences in $\mathrm{TH}$ neurons $(\mathrm{min} / \mathrm{max}=1 \% / 14 \%)$. Although the proportion of reliable sequences in the NR and mPFC were not significantly different (Kolmogorov-Smirnov test, $p=0.15$ ), the average Spearman correlation values of the reliable sequences were significantly larger in the NR than in the mPFC (KolmogorovSmirnov test, $p<0.01$; Fig. $1 D$ ), indicating that the NR cell assembly sequential organization is the most consistent. Since sequences in NR preceded those of $\mathrm{mPFC}$, and reliable sequences can be found in both, we checked whether the reliability in NR could affect that of mPFC. To do so, we compared the reliability index of NR and mPFC within the same UP state in the $n=3$ paired recordings. We found a very small correlation between the two reliability indices (median $r: 0.09 \mathrm{~min} / \mathrm{max}: 0.06 / 0.12$ ). To estimate the participation of neurons in sequence generation, we calculated a participation index, defined as the probability for a neuron to be involved in a given sequence (see Materials and Methods). The average participation index was consistently larger across experiments in the NR (median: $0.63, \mathrm{~min} / \mathrm{max}$ : $0.55 / 0.81$ ) than in the mPFC (median: 0.52 , min/max: $0.36 / 0.60$; Kolmogorov-Smirnov test, $p<0.05$ ). We also calculated the average fraction of nonparticipating neurons (not fulfilling the condition of the robust fit, see Materials and Methods) and compared this with the fraction of nonparticipating neurons because of the nonfiring condition. A very small fraction of neurons was classified as nonparticipating from the outlier condition (median $2.5 \%$ in NR and $1.4 \%$ in $\mathrm{mPFC}$ ) compared with the nonfiring condition (median $30.2 \%$ in NR and $46.0 \%$ in $\mathrm{mPFC}$ ).

As a cross-validation, to discard the possibility that the sequential ordering was because of random fluctuations, we compared the average preferred phases using the first half of the spike train with the average phases using the second half (Fig. 2A). Both for $\mathrm{NR}$ and $\mathrm{mPFC}$, the average phases obtained in the two halves were strongly correlated (median $r: 0.94, \mathrm{~min} / \mathrm{max}$ : 0.8/0.97).

The sequential firing of neurons could just be the result of an increased excitability of early firing neurons compared with neurons firing later after the onset of the UP state (Peyrache et al.,
2010). To address this issue, we recalculated the average phases and the subsequent template ordering by normalizing all the neurons' spike trains via random spike removal to have an average firing rate of $2 \mathrm{~Hz}$. Average phases obtained with the normalized and unnormalized spike trains were virtually identical in NR and mPFC ( $r \sim 1$ aggregated data; Fig. $2 B$ ). Additionally, we tested the correlation between the firing order and the firing rate for each NR and mPFC neuron. We found that 2 of $5 \mathrm{NR}$ recordings were significantly correlated while only 1 of $7 \mathrm{mPFC}$ recordings showed significant correlations (Spearman test, $p<0.05$ ).

Together, these results show that MPFC and NR neurons display highly stable patterns of sequential neuronal activations at the onset of the UP state, with NR sequences being more reproducible. The sequential activation cannot be solely explained by increased excitability of early firing cells.

\section{NR cell assemblies are spatially distributed}

We also investigated the spatial distribution of the NR and mPFC cell assemblies. To do this, we correlated the template order of each neuron to their anatomic dorsoventral localization, since the silicon probes were sampling NR from the dorsal-toventral pole (Fig. $3 A$ ). The slope of a linear fit of such correlation provides the information on whether the activity is propagating in a given direction (Luczak et al., 2007) (see Materials and Methods; Fig. $3 B$ ). Furthermore, we quantified the degree of spatial distribution of the activity for each UP state, calculating a linear fit between the activation latency of each neuron relative to the peak activity and the dorsoventral localization. The slopes of the fit for each UP state, properly multiplied by the number of sites of the probe, resulted in the sequence delay (see Materials and Methods). Positive or negative delays are fingerprints of propagating activity in a given direction. Although both NR and mPFC can show some degree of propagation in a preferential direction (Fig. 3C), NR average delays were consistently larger than $\mathrm{mPFC}$ ones (Kolmogorov-Smirnov test, $p<0.05$; Fig. $3 D$ ), reflecting a dorsal-to-ventral direction of the sequential activation. To assess the consistency of the preferential direction of propagation, we calculated the Spearman rank correlation between the rank of the template order and the anatomic location of the corresponding recording site. We found a highly significant correlation in NR recordings in most cases $(n=4$ of 5 , median $|r|$ value across all $n: 0.65, p<0.001)$, whereas it was virtually absent in $\operatorname{mPFC}(n=1$ of $7,|r|=0.35$, $p<0.01)$. There are thus more spatially organized sequences in NR compared with $\mathrm{mPFC}$ ( $z$ test, $p<0.05$; Fig. $3 E$ ).

Together, these results demonstrate that NR displays at the UP state onset robust sequential activations, which are spatially organized in a dorsoventral pattern. This is particularly surprising as it suggests a topical spatial organization in the NR.

\section{NR firing is necessary to the $\mathrm{MPFC}$ and HPC sequence stability}

The fact that NR cell assemblies occur earlier than mPFC ones raises the possibility that the NR may be involved in the sequential activation of mPFC neurons. To test this hypothesis, we used muscimol to inactivate chemically the NR (see Materials and Methods) and recorded the activity of $\mathrm{mPFC}$ neurons $(n=190$ neurons, $n=5$ experiments). Following NR inactivation, $\mathrm{mPFC}$ neurons showed a reduced firing activity during the UP state compared with noninactivation, control recordings (Fig. 4A) (Ferraris et al., 2018). During SOs, the distribution of interspike intervals is characterized by the presence of two peaks: one at $\sim 1 \mathrm{~Hz}$ corresponding to the frequency of the SO, and one at 
A

NR

mPFC

HPC

Mean Phase
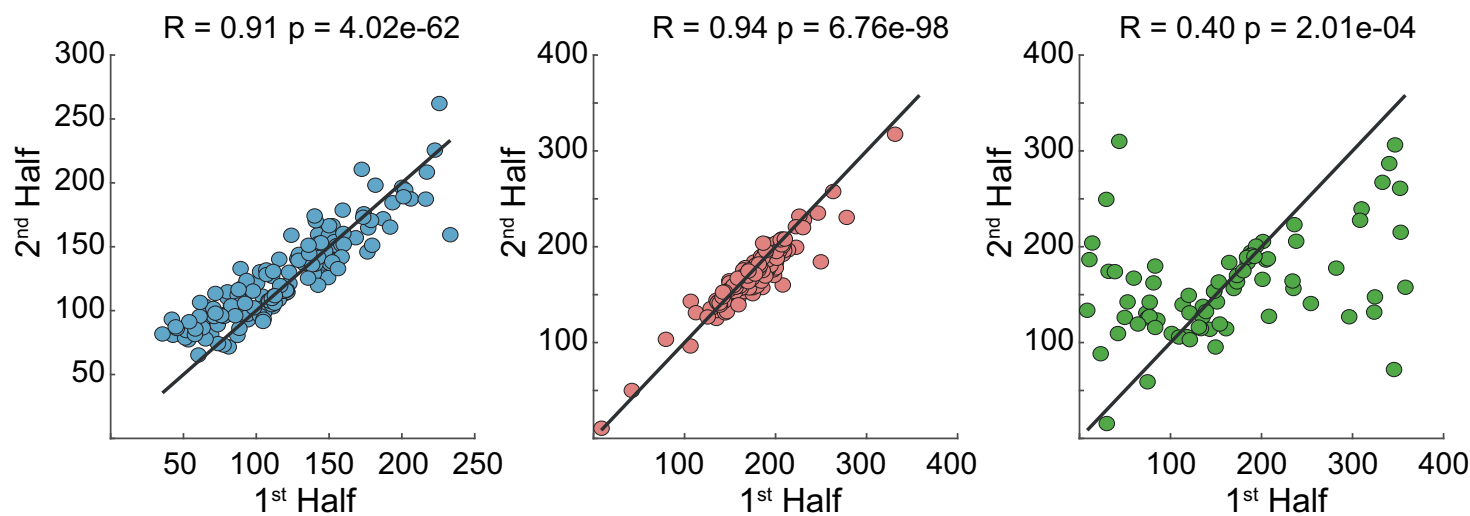

Mean Latency (center of mass)
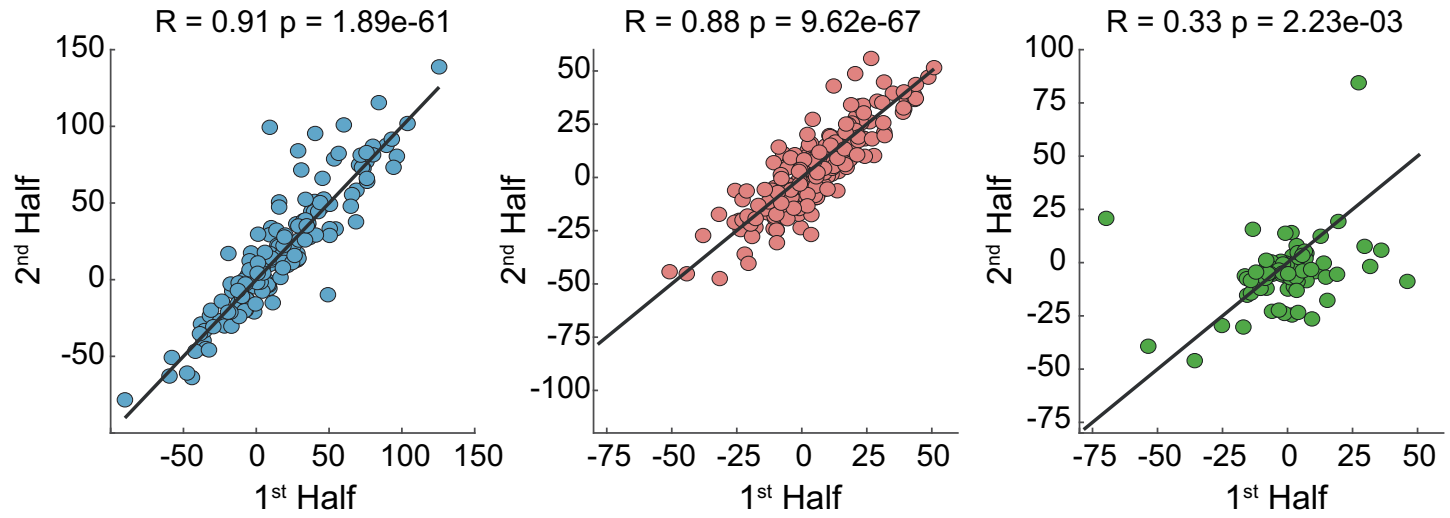

B

NR $r=0.99, p=1.96 e-92$

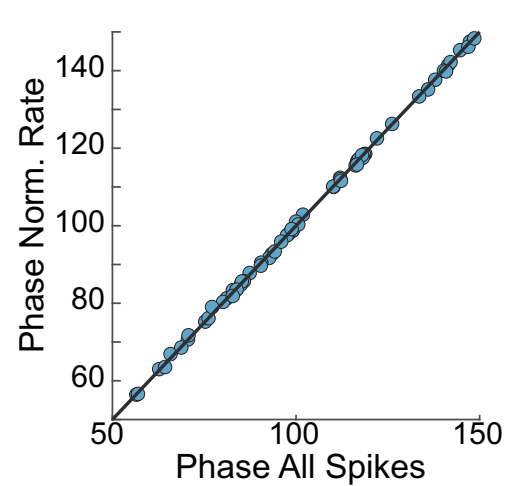

MPFC $r=0.99, p=1.59 e-206$

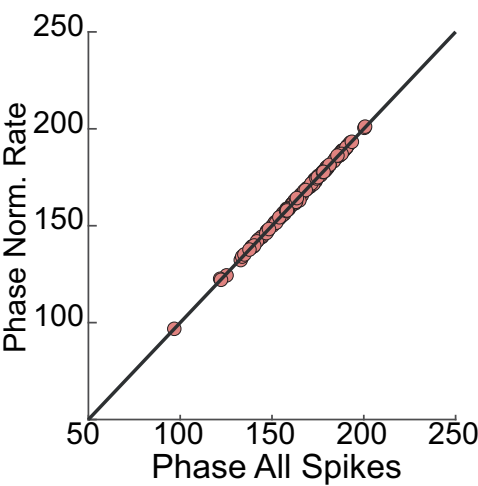

TH $r=0.99, p=1.07 e-47$

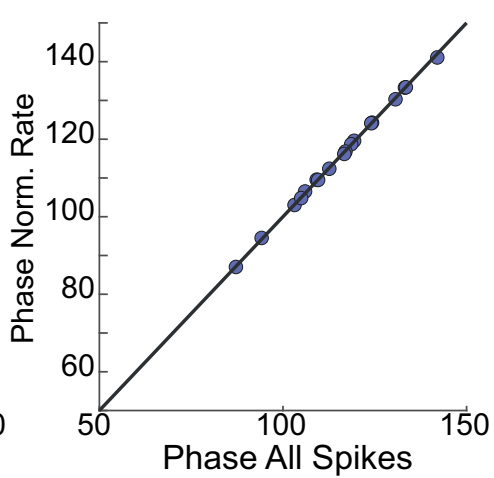

Figure 2. Cross-validations of the sequential dynamics of cell assemblies. $A$, Top, Average phases calculated separately during the first half and second half of the recordings for each region. Bottom, Same as top panels using the average latency method. Both methods lead to similar results. $\boldsymbol{B}$, Average firing phases of NR, mPFC, and TH neurons with reference to the SO calculated using the whole spike train versus the normalized firing rate $(2 \mathrm{~Hz})$. The lack of difference indicates that the sequential activity does not appear to be caused by a mere increase of excitability.

$\sim 100 \mathrm{~ms}$, which indicates that cells fire bursts of action potentials (Fig. 4B). The distribution of interspike intervals was modified in inactivation conditions with a smaller peak at $\sim 100 \mathrm{~ms}$, reflecting reduced bursting activity during UP state (Fig. $4 B$ ). We then calculated the distribution of the pooled variability of the APTH (see Materials and Methods; Fig. 4C, inset) in control and NR inactivation conditions. The median APTH variability across experiments was not significantly changed when NR was inactivated (control median: 9.62, $\mathrm{min} / \mathrm{max}$ : 8.98/10.60, NR inactivation median: 9.02, $\mathrm{min} / \mathrm{max}$ : 8.67/13.73, Kolmogorov-Smirnov test, $p=0.4428$ ), as shown in Figure $4 C$. This reveals the fact that, although bursting is reduced, the time extent to which neurons in $\mathrm{mPFC}$ fire is maintained. However, the UP state duration was slightly shorter (control: $0.55 \mathrm{~s}$, NR inactivation: $0.48 \mathrm{~s}$, 
A

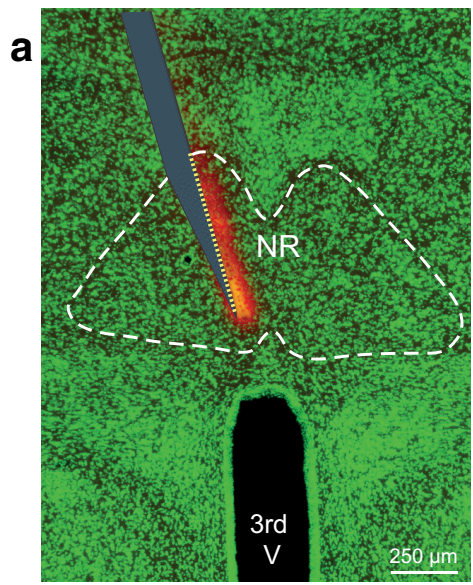

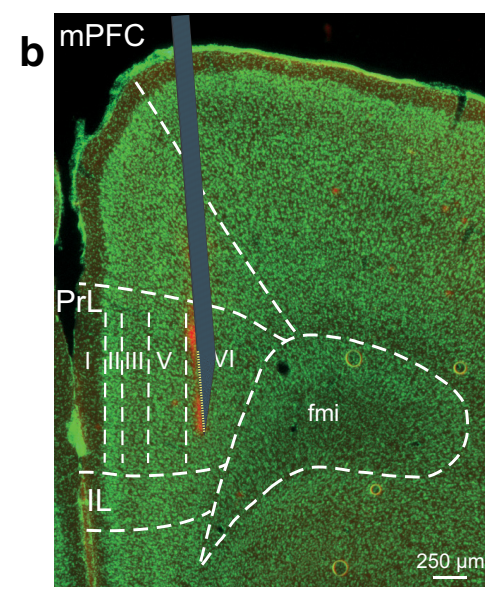

Nis

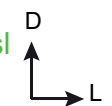

B
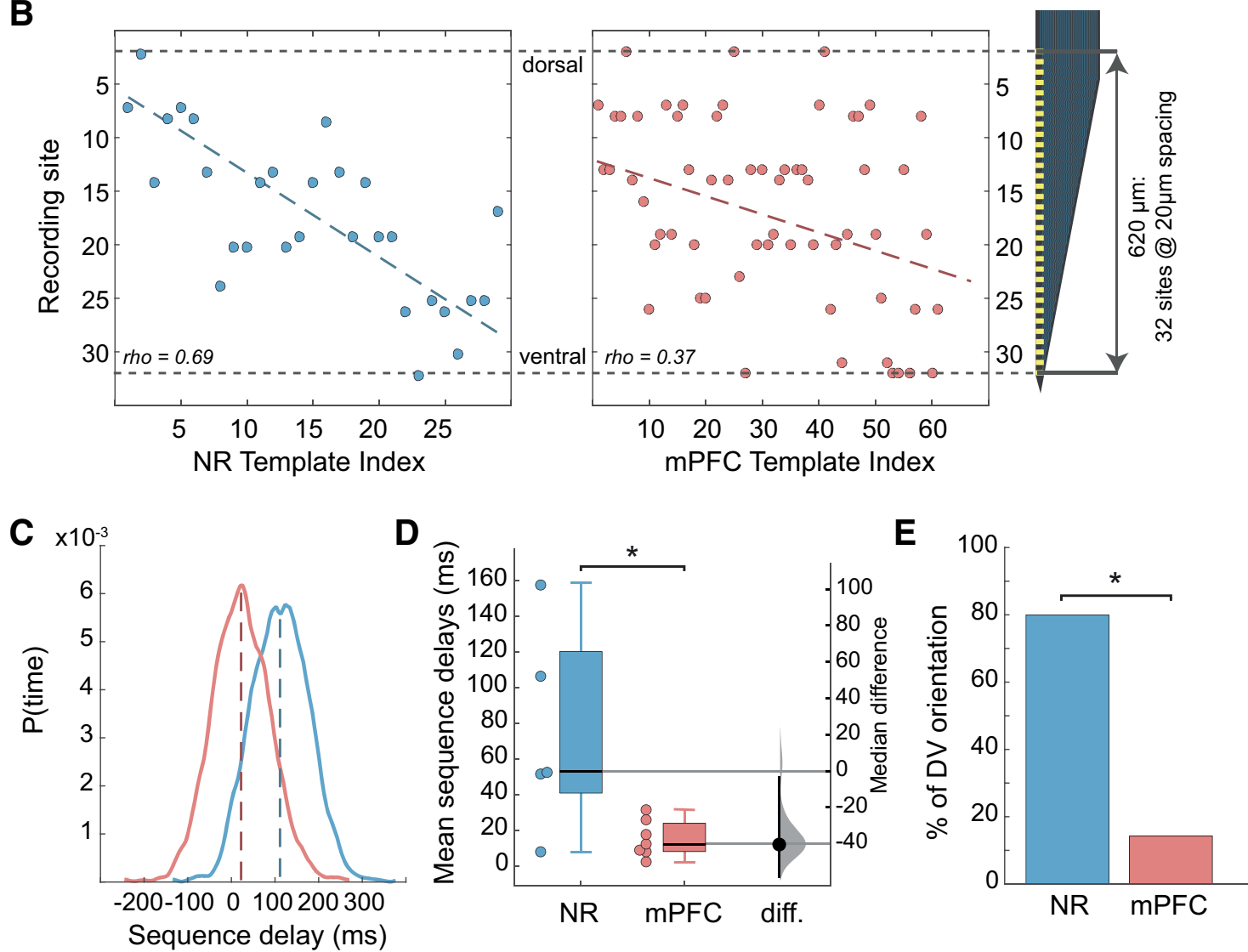

Figure 3. Spatial organization of NR and mPFC cell assemblies. $\boldsymbol{A}$, Representation of the position of a linear silicon probe with 32 recording sites in ( $\boldsymbol{A} \boldsymbol{a})$ the NR and ( $\boldsymbol{A} \boldsymbol{b})$ mPFC. NR and mPFC contours (and layers) are delimited by the white dashed line over the green fluorescent Nissl staining. fmi, Forceps minor of the corpus callosum; third V, third ventricle; PrL, prelimbic area; IL, infralimbic area. Red-orange staining represents the Dil that was deposited at the back of the silicon probe before insertion. D, Dorsal; L, lateral. $B$, Relationship between anatomic dorsoventral location of NR (left) and MPFC (right) neurons (location defined by the site of the probe recording the maximum amplitude of the action potentials) and the template rank showing a linear correlation for NR neurons but not for mPFC ones in a template experiment. C, Distribution of spatial delays (from the beginning to end of a sequence) across the probe from the topmost site to the bottom-most one for mPFC (red) and NR (blue) neurons. D, Mean sequence delays (with median difference and $95 \% \mathrm{Cl}$ ) across experiments for NR and mPFC. $\boldsymbol{E}$, The percentage of experiments showing a dorsoventral (DV) orientation of sequences was larger in the NR than in the mPFC. ${ }^{*} p<0.05$ ).

Mann-Whitney test, $p<0.001$ ), an effect that can be attributed to the extra peak in UP state duration distribution at $\sim 0.25 \mathrm{~s}$, which did not exist in control data (Fig. $4 D$ ). We then evaluated the outcome of the mPFC sequences in the NR inactivation condition. Inhibition of NR activity reduced the capacity of mPFC to generate reliable sequences compared with the control condition, since only $5.6 \%$ of them $(\min / \max : 1.4 \% / 8.4 \%)$ were reliable (compared with control median: 25\%, min/max: 5\%/ $30 \%$, Kolmogorov-Smirnov test, $p=0.0228$; Fig. $4 E$ ). Moreover, the fraction of participating neurons in reliable sequences decreased in the inactivation condition (control: 53\%; inactivation: 37\%; Kolmogorov-Smirnov test, $p<1 \mathrm{e}-5$; Fig. $4 F$ ). These results support the proposal that NR activity is important for the stability and reliability of mPFC sequences. 


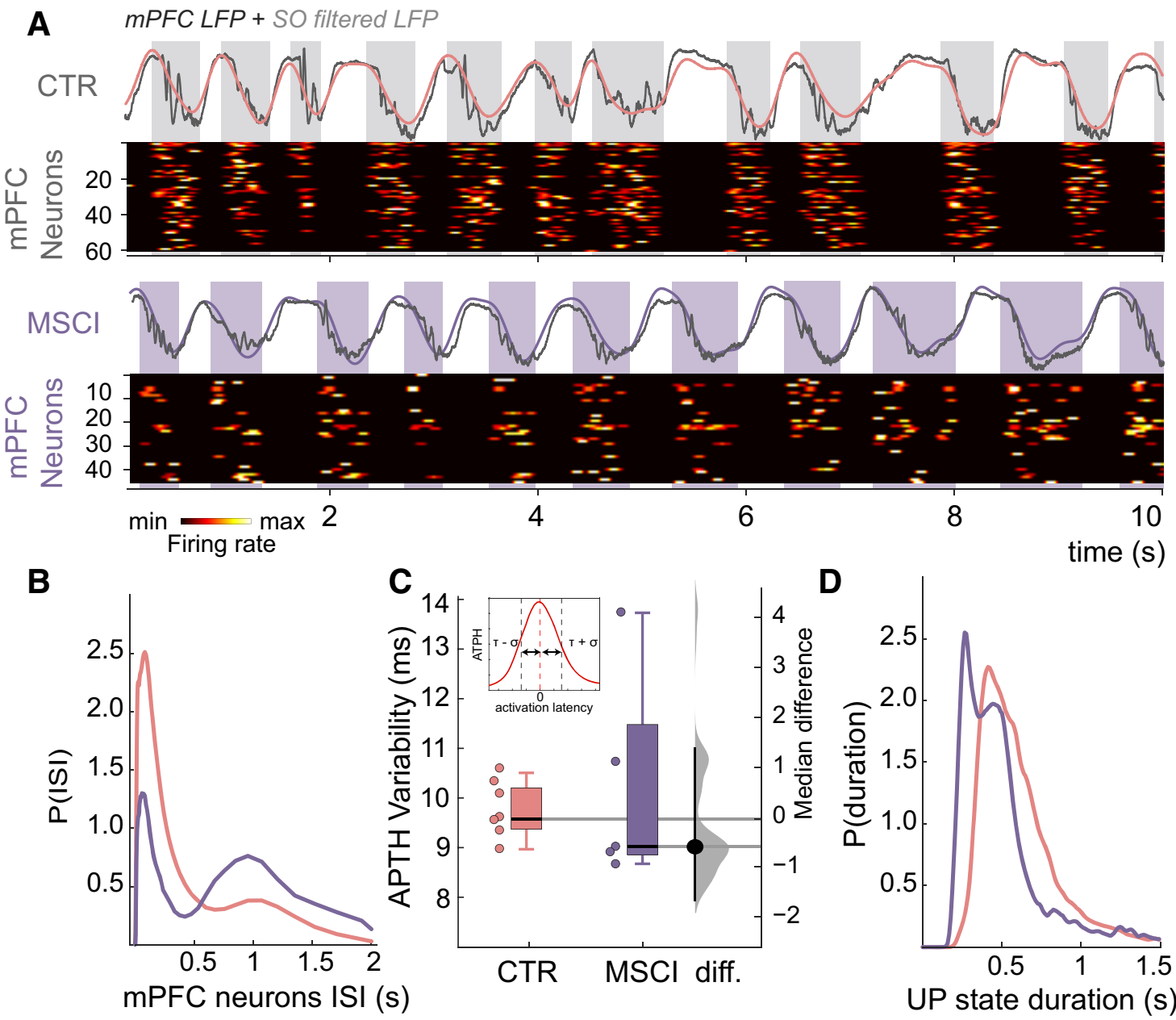

E

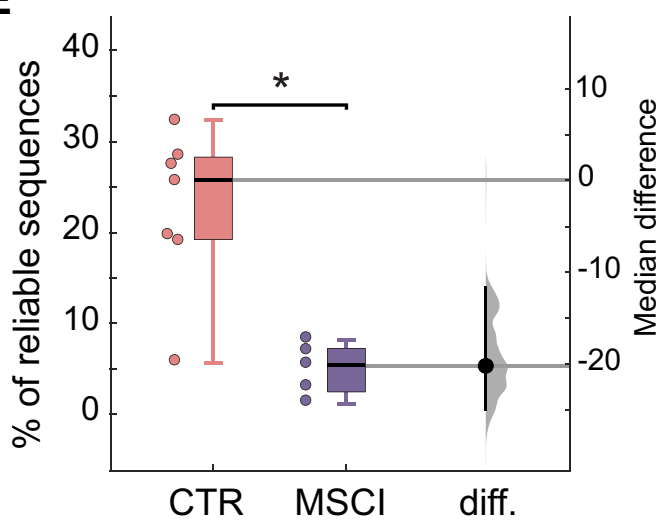

$\mathbf{F}$

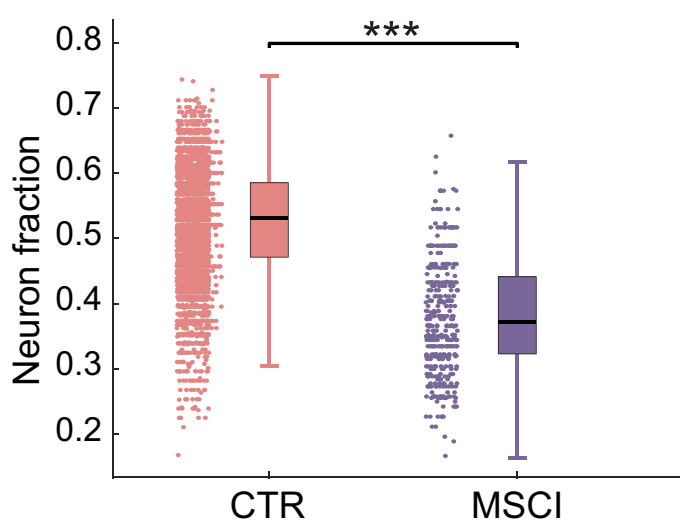

Figure 4. Inactivation of NR impairs reliable sequential activation of mPFC neurons at the beginning of the UP state. $\boldsymbol{A}$, Template heat maps of normalized $m P F C$ unit activities during NR inactivation condition (bottom) inducing less stable cell assemblies compared with control condition (top). The mPFC LFP appears above the heat maps in gray with the corresponding [0.5 2] $\mathrm{Hz}$ filtered trace (pink and purple), and the UP states marked by the gray and purple boxes. $\boldsymbol{B}$, Interspike interval distribution of mPFC neurons grouped data for control (pink) and NR inactivation conditions (purple), showing that a decrease in burst firing when the NR is inactivated. C, Grouped data with median difference and $95 \%$ Cl of the variability of the APTH showing no significant change between control (CTR) and NR inactivation conditions (MSCI). Inset, The APTH quantifies the lag distribution with respect to the population activity peak for each neuron. $\boldsymbol{D}$, Distribution of the duration of the UP state in control (pink) and NR inactivation (purple) condition, where a peak at short values indicates the emergence of shorter UP states. $\boldsymbol{E}$, The number of reliable sequences found in mPFC was decreased when the NR was inactivated (MSCl) compared with the control (CTR) condition (grouped data, $n=5$ experiments). $F$, The fraction of mPFC neurons participating to reliable sequences was decreased when the NR was inactivated. $\left({ }^{*} p<0.05,{ }^{* * *} p<0.001\right)$.

The UP state of the SOs poorly modulates the activity of HPC neurons and the formation of timely organized cell assemblies. However, sequential activation of HPC neurons consistently occurs during SPW-Rs (Buzsáki, 2015). We therefore investigated the consequences of NR inactivation on HPC sequences during SPW-Rs. NR inactivation did not affect HPC neurons firing rate (control average: $1.07 \pm 0.05 \mathrm{~Hz}$, NR inactivation average: $1.08 \pm 0.05 \mathrm{~Hz}, t$ test, Cohen's $d=0.019, p=0.97$; Fig. $5 A, E$ ). In addition, NR inactivation did not alter the frequency of SPW$\mathrm{R}$ occurrence (median control: $0.087 \mathrm{~Hz}, \min / \mathrm{max}$ : 0.043 / 


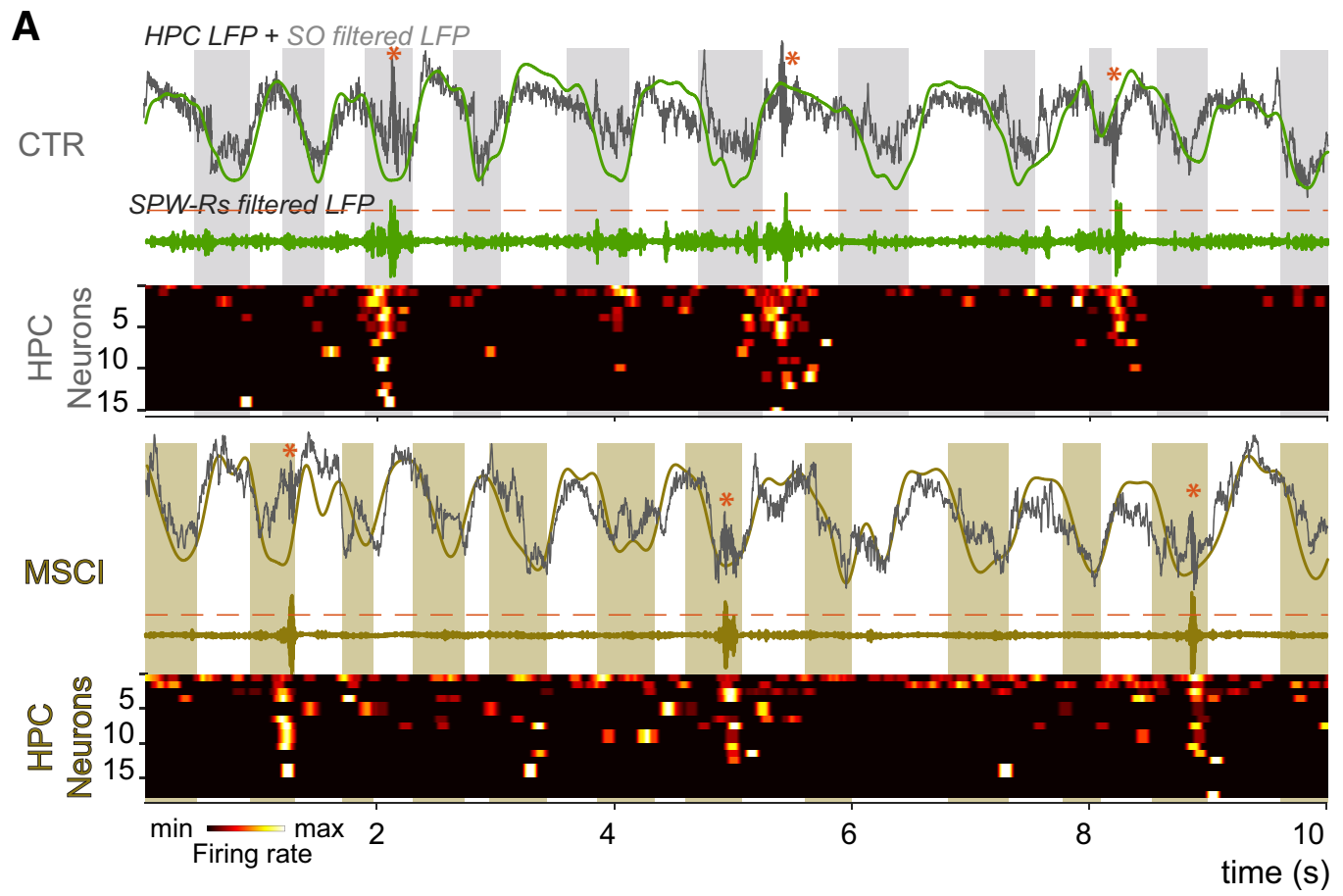

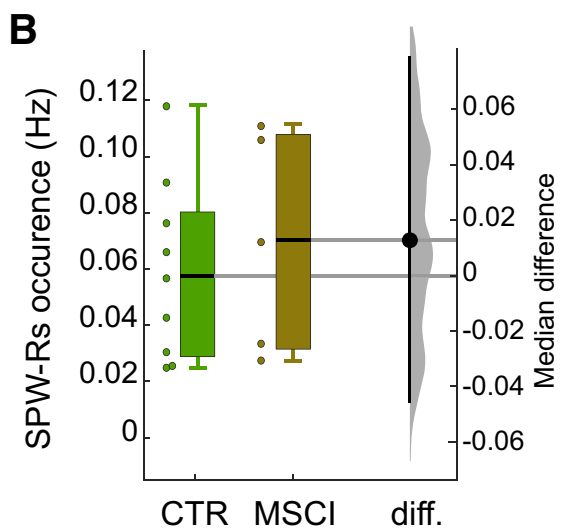

E

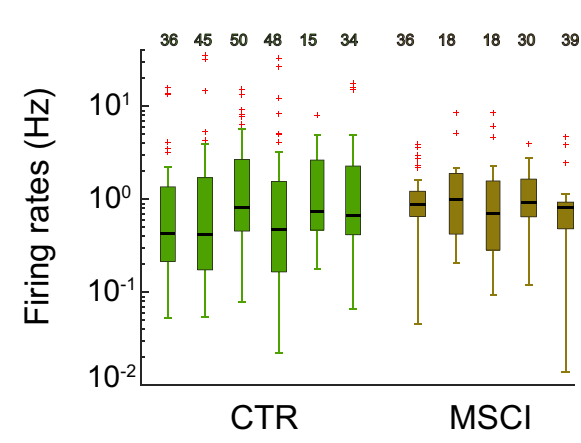

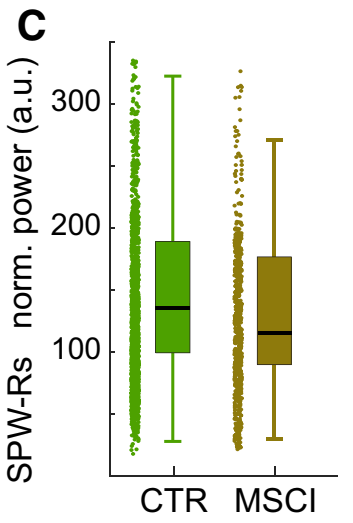

$\mathbf{F}$

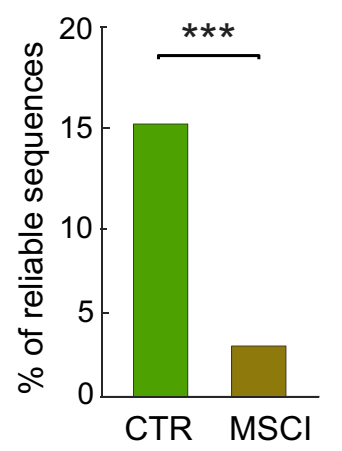

D

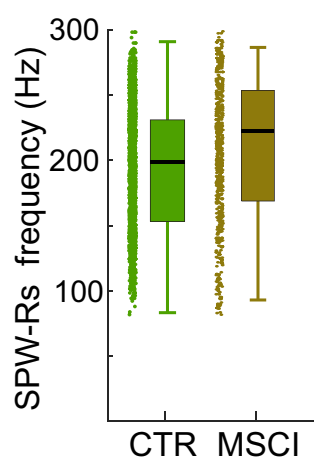

G

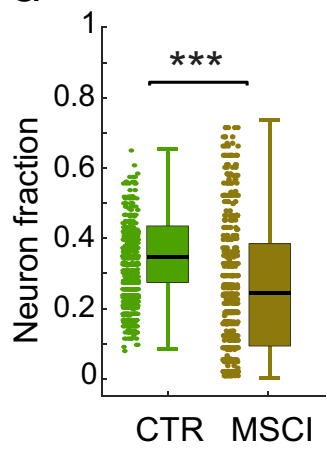

Figure 5. Inactivation of NR impairs reliable sequential activation of HPC neurons during SPW-Rs. A, Template heat maps of normalized HPC unit activities in control (CTR) and NR inactivation (MSCI) condition (bottom of each panel). Top, The HPC LFP (gray) and the corresponding [0.5 2] Hz filtered trace (green and kaki). Gray and Khaki boxes represent the UP states. Orange stars represent the SPW-Rs, which are visible in the [80 200] Hz filtered LFP (bottom). B, SPW-R occurrence did not change when NR was inactivated (grouped data, $n=5$ experiments; $n=913$ SPW-R events in (TR, $n=149$ SPW-R events in MSCI). C, NR inactivation did not change the normalized power (a.u., Arbitrary unit) or the (D) inner frequency of SPW-Rs. $\boldsymbol{E}$, The median firing rates of HPC neurons were not affected by NR inactivation. Red crosses represent outlier values. The numbers on the top of each statistical box indicate the number of recorded neurons in the session. $\boldsymbol{F}$, The proportion of reliable cell assemblies during SPW-Rs was considerably reduced when the NR was inactivated. $\mathbf{G}$, The fraction of reliably participating HPC neurons to sequences during SPW-R events was decreased by NR inactivation. ( $\left.{ }^{* *} p<0.001\right)$. 
$0.11 \mathrm{~Hz}, \mathrm{NR}$ inactivation: $0.049 \mathrm{~Hz}, \min / \max : 0.022 / 0.111 \mathrm{~Hz}$, Kolmogorov-Smirnov test, $p=0.8254$; Fig. $5 B$ ). Similarly, neither their power (mean normalized power control: $157 \pm 2$, NR inactivation: $144 \pm 5, t$ test, $p=0.295$; Fig. $5 C$ ) nor their inner frequency (mean frequency control: $193 \pm 1 \mathrm{~Hz}$, NR inactivation: $213 \pm 3 \mathrm{~Hz}, t$ test, $p=0.084$; Fig. $5 D$ ) was modified. This result shows that NR inactivation does not change either SPW-R properties or global HPC cell firing. In contrast, the number of reliable sequences found within SWP-Rs was largely reduced when the NR was inactivated (control median: $15 \%$, NR inactivation median: $3 \%, z$ test, $p<0.001$; Fig. $5 F$ ). In this condition, the remaining cell assemblies recruited a significantly lower number of neurons (control median: 37.5\%, min/max: 12.5\%/80\%, NR inactivation median: $27.8 \%$, $\mathrm{min} / \mathrm{max}$ : $0 \% / 74.4 \%$, KolmogorovSmirnov test, $p<0.001$; Fig. $5 G$ ). These findings suggest that the $\mathrm{NR}$ activity has a causal role in the sequential organization of neuronal firing in the HPC during SPW-Rs.

\section{Discussion}

In this study, we show that four-fifths of NR neurons fire within robustly spatially and temporally organized cell assemblies at UP state onset; and that NR activity influences cell assemblies' stability in $\mathrm{mPFC}$ at UP state onset and in HPC during SPW-Rs. These results further support the concept that the NR is a key functional hub in memory networks involving the MPFC and the HPC.

The sequential activation of neuronal assemblies is believed to constitute a core feature of information processing in the brain (Hebb, 1949; Buzsáki, 2010). Cell assemblies are found in the archicortex (Lee and Wilson, 2002; Harris et al., 2003; Dragoi and Buzsáki, 2006; Pastalkova et al., 2008; Villette et al., 2015; Malvache et al., 2016), cortical areas (Kenet et al., 2003; MacLean et al., 2005; Ferezou et al., 2006; Euston et al., 2007; Luczak et al., 2007; Luczak and Maclean, 2012), as well as in striatum (Lansink et al., 2009). They constitute a way to code/encode/store information (Maass, 2016; Kitamura et al., 2017). During NREM sleep, cortical activity is dominated by the sequential activation of cortical neurons at the onset of the UP state, while in the HPC the sequential activation mostly occurs during SPW-Rs (Sirota et al., 2003; Battaglia et al., 2004; Peyrache et al., 2009; Maingret et al., 2016; Khodagholy et al., 2017). Similar sequential firing occurs during the SOs measured during anesthesia (Luczak et al., 2007), supporting the view that such brain state shares many features with NREM sleep (Tung and Mendelson, 2004; Isomura et al., 2006; Clement et al., 2008; Quilichini et al., 2010; Hutt, 2011; Ferraris et al., 2018). Whether sequences represent internally generated representations (Pastalkova et al., 2008), preconfigured cell assemblies (Dragoi and Tonegawa, 2011; Liu et al., 2019) or a functional template of offline replay in the framework of memory consolidation (Lee and Wilson, 2002; Buzsáki, 2015; Pfeiffer, 2020) still remains to be elucidated. Our work demonstrates that sequences can also be recorded in a thalamic nucleus. This property appears to be specific to the NR, on the basis of recordings of other thalamic nuclei recorded in the vicinity. A systematic study of all thalamic nuclei should now be performed, to determine whether this property is specific to the NR. In the present work, we used anesthesia conditions; therefore, our results should also be validated during long sleep sessions. It is, however, noteworthy that anesthesia is likely to affect the network dynamics and neuronal firing. As shown by Luczak et al. (2007), anesthesia provides a regular alternation of UP and DOWN, whereas natural sleep displays much briefer DOWN states, resulting in highly asymmetric field events. They, however, show that neuronal firing patterns at UP state onset bear clear similarities between anesthesia and natural sleep, arguing that the results obtained here are informative about natural brain dynamics.

The way $\mathrm{mPFC}$ and HPC sequences are generated remains poorly understood. Our results demonstrate that NR activity is a key regulator of $\mathrm{mPFC}$ and HPC sequence stability. Interestingly, previous (Ferraris et al., 2018) and present results demonstrate that the NR does not seem to regulate oscillatory patterns, as NR inactivation does not change the frequency of SOs, $\gamma$ oscillations, and SPW-Rs. It is yet important to note that our NR manipulations indicate a causal role for NR activity, but not necessarily for the sequential organization of its neurons. However, the NR activity appears to organize the synchronization of $\gamma$ oscillations between the HPC and the mPFC (Ferraris et al., 2018) as well as the sequential activation of cell assemblies in the MPFC during SOs or in the HPC during SPW-Rs. The NR is ideally located for such fine-tuning of cell activity, as it is bidirectionally connected to the mPFC and HPC (Vertes, 2006; Cassel et al., 2013; Varela et al., 2014). The fact that NR sequences always precede mPFC ones at UP state onset suggests that NR cells may directly drive mPFC cells. In keeping with this proposal, NR neurons have an excitatory action on HPC and mPFC by modulating/activating both interneurons and principal cells (Dolleman-Van der Weel et al., 1997; Dolleman-Van der Weel and Witter, 2000; Di Prisco and Vertes, 2006), their axons innervating the stratum lacunosum moleculare of CA1 of mainly the ventral and intermediate portions of the HPC (Wouterlood et al., 1990; Vertes et al., 2006), and the infralimbic, prelimbic, and anterior cingulate cortices of the mPFC with fibers terminating densely in layer 1 and layers 5/6 of these regions (Hoover and Vertes, 2012; Varela et al., 2014). However, the influence of NR activity on sequences during hippocampal SPW-Rs is more difficult to explain as SPW-Rs occur at variable times after UP state onset (Sirota et al., 2003; Battaglia et al., 2004). This rather suggests that the NR is an important hub in a wider network of networks, from which oscillations can emerge.

The presence of assemblies specifically in the NR is quite remarkable. Since NR neurons are involved in reference memory consolidation (Loureiro et al., 2012) and in spatial memory (Jankowski et al., 2014, 2015; Ito et al., 2015; Cholvin et al., 2018), NR assemblies may constitute an activity template used to organize information at the beginning of the UP state. Such a mechanism may enable the transmission of information to target areas in a packet-based manner (Luczak and Maclean, 2012), a default activity in a default mode (Sanchez-Vives and Mattia, 2014; Luczak et al., 2015).

Another remarkable feature of NR assemblies is their dorsoventral organization, which suggests a precise topological organization in terms of afferences and efferences despite the fact that NR does not have a layered organization (Jones, 1985; Bokor et al., 2002; Van der Werf et al., 2002; Dolleman-van der Weel et al., 2019). The NR probe was inserted in the brain with an angle to properly target the NR (Fig. 3Aa). As a result, the bottommost sites were located more medially than the top-most ones. Although the mediolateral distance was $<250 \mu \mathrm{m}$ between the top and bottom sites, we cannot fully rule out that the spatial organization we describe is mediolateral rather than dorsoventral (or both). Luczak et al. (2007) have shown that local sequences in the cortex are independent of global activity patterns, such as traveling waves, which argues in favor of local computation. However, the NR sequences described here are identified within a smaller spatial scale $(<250 \times 620 \mu \mathrm{m}$ here vs $160 \times 1400 \mu \mathrm{m}$ in Luczak et al. (2007)), indicating that those NR sequences might lie somewhere between entities of global patterns and of local 
networks. The origin of the dorsoventral organization of these sequences remains to be elucidated. While NR outputs to the subiculum are topographically organized along the dorsoventral axis, no study has reported such precise topological organization of NR inputs (Dolleman-Van Der Weel and Witter, 1996). Only differences involving the rostrocaudal axis have been so far reported (Cassel et al., 2013), as well as a preferential targeting of NR fibers to prelimbic and infralimbic deep layers (5 and 6) and superficial layer 1 in mPFC (Berendse and Groenewegen, 1991; Vertes, 2004; Hoover and Vertes, 2012). The fact that we could not find any dorsoventral pattern in mPFC sequences does not mean that there is none to be found as, given the spatial organization of the mPFC, we could only target one layer. There is also no available information on the local connectivity among NR neurons, except a caudal to rostral pathway (Dolleman-Van der Weel et al., 1997). The NR includes difference cell types, but the lack of specific molecular markers prevents, so far, a proper optogenetic investigation (Bokor et al., 2002; Walsh et al., 2017; Dolleman-van der Weel et al., 2019).

In conclusion, our results further support the concept that the NR plays a key role as an anatomic and functional hub between the $\mathrm{mPFC}$ and the HPC. The influence it exerts on mPFC and HPC information packets suggests that it strongly participates in the organization of information in both regions but also in the transfer of information from the HPC to the mPFC. Its internal organization allows the genesis of information packet sequences, which may represent similar features as those coded in the $\mathrm{mPFC}$ and the HPC.

\section{References}

Atherton LA, Dupret D, Mellor JR (2015) Memory trace replay: the shaping of memory consolidation by neuromodulation. Trends Neurosci 38:560-570.

Battaglia FP, Sutherland GR, McNaughton BL (2004) Hippocampal sharp wave bursts coincide with neocortical "UP state" transitions. Learn Mem 11:697-704.

Battaglia FP, Benchenane K, Sirota A, Pennartz CM, Wiener SI (2011) The hippocampus: hub of brain network communication for memory. Trends Cogn Sci 15:310-318.

Belluscio MA, Mizuseki K, Schmidt R, Kempter R, Buzsáki G (2012) Crossfrequency phase-phase coupling between theta and gamma oscillations in the hippocampus. J Neurosci 32:423-435.

Berendse HW, Groenewegen HJ (1991) Restricted cortical termination fields of the midline and intralaminar thalamic nuclei in the rat. Neuroscience 42:73-102.

Berens P (2009) CircStat: a MATLAB toolbox for circular statistics. J Stat Softw 31:1-21.

Bokor H, Csáki A, Kocsis K, Kiss J (2002) Cellular architecture of the nucleus reuniens thalami and its putative aspartatergic/glutamatergic projection to the hippocampus and medial septum in the rat. Eur J Neurosci $16: 1227-1239$

Buzsaki G (2019) The brain from inside out. Oxford: Oxford UP.

Buzsáki G (2010) Neural syntax: cell assemblies, synapsembles, and readers. Neuron 68:362-385.

Buzsáki G (2015) Hippocampal sharp wave-ripple: a cognitive biomarker for episodic memory and planning. Hippocampus 25:1073-1188.

Buzsáki G, Draguhn A (2004) Neuronal oscillations in cortical networks. Science 304:1926-1929.

Buzsáki G, Schomburg EW (2015) What does gamma coherence tell us about inter-regional neural communication? Nat Neurosci 18:484-489.

Buzsáki G, Anastassiou CA, Koch C (2012) The origin of extracellular fields and currents: EEG, ECoG, LFP and spikes. Nat Neurosci 13:407-420.

Calin-Jageman RJ, Cumming G (2019) Estimation for better inference in neuroscience. eNeuro 6:ENEURO.0205-19.2019.

Cassel JC, Pereira de Vasconcelos A, Loureiro M, Cholvin T, DalrympleAlford JC, Vertes RP (2013) The reuniens and rhomboid nuclei: neuroanatomy, electrophysiological characteristics and behavioral implications. Prog Neurobiol 111:34-52.
Cholvin T, Hok V, Giorgi L, Chaillan FA, Poucet B (2018) Ventral midline thalamus is necessary for hippocampal place field stability and cell firing modulation. J Neurosci 38:158-172.

Clement EA, Richard A, Thwaites M, Ailon J, Peters S, Dickson CT (2008) Cyclic and sleep-like spontaneous alternations of brain state under urethane anaesthesia. PLoS One 3:e2004.

Davidson TJ, Kloosterman F, Wilson MA (2009) Hippocampal replay of extended experience. Neuron 63:497-507.

Di Prisco GV, Vertes RP (2006) Excitatory actions of the ventral midline thalamus (rhomboid/reuniens) on the medial prefrontal cortex in the rat. Synapse 60:45-55.

Dolleman-Van Der Weel MJ, Witter MP (1996) Projections from the nucleus reuniens thalami to the entorhinal cortex, hippocampal field CA1, and the subiculum in the rat arise from different populations of neurons. J Comp Neurol 364:637-650.

Dolleman-Van der Weel MJ, Witter MP (2000) Nucleus reuniens thalami innervates gamma aminobutyric acid positive cells in hippocampal field CA1 of the rat. Neurosci Lett 278:145-148.

Dolleman-Van der Weel MJ, Lopes da Silva FH, Witter MP (1997) Nucleus reuniens thalami modulates activity in hippocampal field CA1 through excitatory and inhibitory mechanisms. J Neurosci 17:5640-5650.

Dolleman-van der Weel MJ, Griffin AL, Ito HT, Shapiro ML, Witter MP, Vertes RP, Allen TA (2019) The nucleus reuniens of the thalamus sits at the nexus of a hippocampus and medial prefrontal cortex circuit enabling memory and behavior. Learn Mem 26:191-205.

Dragoi G, Buzsáki G (2006) Temporal encoding of place sequences by hippocampal cell assemblies. Neuron 50:145-157.

Dragoi G, Tonegawa S (2011) Preplay of future place cell sequences by hippocampal cellular assemblies. Nature 469:397-401.

Eichenbaum H (2017) Prefrontal-hippocampal interactions in episodic memory. Nat Rev Neurosci 18:547-558.

Euston DR, Tatsuno M, McNaughton BL (2007) Fast-forward playback of recent memory sequences in prefrontal cortex during sleep. Science 318:1147-1150.

Ferezou I, Bolea S, Petersen CC (2006) Visualizing the cortical representation of whisker touch: voltage-sensitive dye imaging in freely moving mice. Neuron 50:617-629.

Ferraris M, Ghestem A, Vicente AF, Nallet-Khosrofian L, Bernard C, Quilichini PP (2018) The nucleus reuniens controls long-range hippocampo-prefrontal gamma synchronization during slow oscillations. J Neurosci 38:3026-3038.

Foster DJ, Wilson MA (2006) Reverse replay of behavioural sequences in hippocampal place cells during the awake state. Nature 440:680-683.

Frankland PW, Bontempi B (2005) The organization of recent and remote memories. Nat Rev Neurosci 6:119-130.

Harris KD, Henze DA, Csicsvari J, Hirase H, Buzsáki G (2000) Accuracy of tetrode spike separation as determined by simultaneous intracellular and extracellular measurements. J Neurophysiol 84:401-414.

Harris KD, Csicsvari J, Hirase H, Dragoi G, Buzsáki G (2003) Organization of cell assemblies in the hippocampus. Nature 424:552-556.

Hazan L, Zugaro M, Buzsáki G (2006) Klusters, NeuroScope, NDManager: a free software suite for neurophysiological data processing and visualization. J Neurosci Methods 155:207-216.

Hebb DO (1949) The organization of behavior. New York: Wiley.

Herkenham M (1978) The connections of the nucleus reuniens thalami: evidence for a direct thalamo-hippocampal pathway in the rat. J Comp Neurol 177:589-610.

Herreras O (2016) Local field potentials: myths and misunderstandings. Front Neural Circuits 10:101-101.

Hoover WB, Vertes RP (2012) Collateral projections from nucleus reuniens of thalamus to hippocampus and medial prefrontal cortex in the rat: a single and double retrograde fluorescent labeling study. Brain Struct Funct 217:191-209.

Hutt A (2011) Sleep and anesthesia: neural correlates in theory and experiment. New York: Springer.

Isomura Y, Sirota A, Ozen S, Montgomery S, Mizuseki K, Henze DA, Buzsáki G (2006) Integration and segregation of activity in entorhinal-hippocampal subregions by neocortical slow oscillations. Neuron 52:871-882.

Ito HT, Zhang S, Witter MP, Moser EI, Moser MB (2015) A prefrontal-thalamo-hippocampal circuit for goal-directed spatial navigation. Nature 522:50-55. 
Jankowski MM, Islam MN, Wright NF, Vann SD, Erichsen JT, Aggleton JP, O'Mara SM (2014) Nucleus reuniens of the thalamus contains head direction cells. eLife 3:e03075.

Jankowski MM, Passecker J, Islam MN, Vann S, Erichsen JT, Aggleton JP, O'Mara SM (2015) Evidence for spatially-responsive neurons in the rostral thalamus. Front Behav Neurosci 9:256-256.

Ji D, Wilson MA (2007) Coordinated memory replay in the visual cortex and hippocampus during sleep. Nat Neurosci 10:100-107.

Jones EG (1985) The thalamus. New York: Plenum.

Kenet T, Bibitchkov D, Tsodyks M, Grinvald A, Arieli A (2003) Spontaneously emerging cortical representations of visual attributes. Nature 425:954-956.

Khodagholy D, Gelinas JN, Buzsaki G (2017) Learning-enhanced coupling between ripple oscillations in association cortices and hippocampus. Science 358:369-372.

Kitamura T, Ogawa SK, Roy DS, Okuyama T, Morrissey MD, Smith LM, Redondo RL, Tonegawa S (2017) Engrams and circuits crucial for systems consolidation of a memory. Science 356:73-78.

Klein MM, Cholvin T, Cosquer B, Salvadori A, Le Mero J, Kourouma L, Boutillier AL, Pereira de Vasconcelos A, Cassel JC (2019) Ventral midline thalamus lesion prevents persistence of new (learning-triggered) hippocampal spines, delayed neocortical spinogenesis, and spatial memory durability. Brain Struct Funct 224:1659-1676.

Lansink CS, Goltstein PM, Lankelma JV, McNaughton BL, Pennartz CM (2009) Hippocampus leads ventral striatum in replay of place-reward information. PLoS Biol 7:e1000173.

Latchoumane CF, Ngo HV, Born J, Shin HS (2017) Thalamic spindles promote memory formation during sleep through triple phase-locking of cortical, thalamic, and hippocampal rhythms. Neuron 95:424-435.

Lee AK, Wilson MA (2002) Memory of sequential experience in the hippocampus during slow wave sleep. Neuron 36:1183-1194.

Liu K, Sibille J, Dragoi G (2019) Preconfigured patterns are the primary driver of offline multi-neuronal sequence replay. Hippocampus 29:275-283.

Loureiro M, Cholvin T, Lopez J, Merienne N, Latreche A, Cosquer B, Geiger K, Kelche C, Cassel JC, Pereira de Vasconcelos A (2012) The ventral midline thalamus (reuniens and rhomboid nuclei) contributes to the persistence of spatial memory in rats. J Neurosci 32:9947-9959.

Luczak A, Maclean JN (2012) Default activity patterns at the neocortical microcircuit level. Front Integr Neurosci 6:30.

Luczak A, Bartho P, Marguet SL, Buzsáki G, Harris KD (2007) Sequential structure of neocortical spontaneous activity in vivo. Proc Natl Acad Sci USA 104:347-352.

Luczak A, McNaughton BL, Harris KD (2015) Packet-based communication in the cortex. Nat Rev Neurosci 16:745-755.

Maass W (2016) Searching for principles of brain computation. Curr Opin Behav Sci 11:81-92.

MacLean JN, Watson BO, Aaron GB, Yuste R (2005) Internal dynamics determine the cortical response to thalamic stimulation. Neuron 48:811-823.

Maingret N, Girardeau G, Todorova R, Goutierre M, Zugaro M (2016) Hippocampo-cortical coupling mediates memory consolidation during sleep. Nat Neurosci 19:959-964.

Malvache A, Reichinnek S, Villette V, Haimerl C, Cossart R (2016) Awake hippocampal reactivations project onto orthogonal neuronal assemblies. Science 353:1280-1283.

Manouze H, Ghestem A, Poillerat V, Bennis M, Ba-M'hamed S, Benoliel JJ, Becker C, Bernard C (2019) Effects of single cage housing on stress, cognitive, and seizure parameters in the rat and mouse pilocarpine models of epilepsy. eNeuro 6:ENEURO.0179-18.2019.

Marre O, Yger P, Davison AP, Frégnac Y (2009) Reliable recall of spontaneous activity patterns in cortical networks. J Neurosci 29:14596-14606.

Nádasdy Z (2000) Spike sequences and their consequences. J Physiol Paris 94:505-524.

Nadasdy Z, Hirase H, Czurko A, Csicsvari J, Buzsáki G (1999) Replay and time compression of recurring spike sequences in the hippocampus. J Neurosci 19:9497-9507.

Newman M (2010) Networks: an introduction. Oxford scholarship

Pastalkova E, Itskov V, Amarasingham A, Buzsaki G (2008) Internally generated cell assembly sequences in the rat hippocampus. Science 321:1322-1327.
Pavlides C, Winson J (1989) Influences of hippocampal place cell firing in the awake state on the activity of these cells during subsequent sleep episodes. J Neurosci 9:2907-2918.

Pereira de Vasconcelos A, Cassel JC (2015) The nonspecific thalamus: a place in a wedding bed for making memories last? Neurosci Biobehav Rev 54:175-196.

Peyrache A, Khamassi M, Benchenane K, Wiener SI, Battaglia FP (2009) Replay of rule-learning related neural patterns in the prefrontal cortex during sleep. Nat Neurosci 12:919-926.

Peyrache A, Benchenane K, Khamassi M, Wiener SI, Battaglia FP (2010) Principal component analysis of ensemble recordings reveals cell assemblies at high temporal resolution. J Comput Neurosci 29:309-325.

Pfeiffer BE (2020) The content of hippocampal "replay." Hippocampus 30:6- 13.

Preston AR, Eichenbaum H (2013) Interplay of hippocampus and prefrontal cortex in memory. Curr Biol 23:R764-R773.

Quilichini P, Sirota A, Buzsáki G (2010) Intrinsic circuit organization and theta-gamma oscillation dynamics in the entorhinal cortex of the rat. J Neurosci 30:11128-11142.

Sanchez-Vives MV, Mattia M (2014) Slow wave activity as the default mode of the cerebral cortex. Arch Ital Biol 152:147-155.

Siapas AG, Wilson MA (1998) Coordinated interactions between hippocampal ripples and cortical spindles during slow-wave sleep. Neuron 21:1123-1128.

Sirota A, Buzsáki G (2005) Interaction between neocortical and hippocampal networks via slow oscillations. Thalamus Relat Syst 3:245-259.

Sirota A, Csicsvari J, Buhl D, Buzsáki G (2003) Communication between neocortex and hippocampus during sleep in rodents. Proc Natl Acad Sci USA 100:2065-2069.

Skaggs WE, McNaughton BL (1996) Replay of neuronal firing sequences in rat hippocampus during sleep following spatial experience. Science 271:1870-1873.

Staresina BP, Ole Bergmann T, Bonnefond M, van der Meij R, Jensen O, Deuker L, Elger CE, Axmacher N, Fell J (2015) Hierarchical nesting of slow oscillations, spindles and ripples in the human hippocampus during sleep. Nat Neurosci 18:1679-1686.

Tung A, Mendelson WB (2004) Anesthesia and sleep. Sleep Med Rev 8:213225.

Van der Werf YD, Witter MP, Groenewegen HJ (2002) The intralaminar and midline nuclei of the thalamus: anatomical and functional evidence for participation in processes of arousal and awareness. Brain Res Brain Res Rev 39:107-140.

Varela C, Kumar S, Yang JY, Wilson MA (2014) Anatomical substrates for direct interactions between hippocampus, medial prefrontal cortex, and the thalamic nucleus reuniens. Brain Struct Funct 219:911-929.

Vertes RP (2004) Differential projections of the infralimbic and prelimbic cortex in the rat. Synapse 51:32-58.

Vertes RP (2006) Interactions among the medial prefrontal cortex, hippocampus and midline thalamus in emotional and cognitive processing in the rat. Neuroscience 142:1-20.

Vertes RP, Hoover WB, Do Valle AC, Sherman A, Rodriguez JJ (2006) Efferent projections of reuniens and rhomboid nuclei of the thalamus in the rat. J Comp Neurol 499:768-796.

Villette V, Malvache A, Tressard T, Dupuy N, Villette V, Malvache A, Tressard T, Dupuy N, Cossart R (2015) Internally recurring hippocampal sequences as a population template of spatiotemporal information article internally recurring hippocampal sequences as a population template of spatiotemporal information. Neuron 88:357-366.

Walsh DA, Brown JT, Randall AD (2017) In vitro characterization of celllevel neurophysiological diversity in the rostral nucleus reuniens of adult mice. J Physiol 595:3549-3572.

Wilson MA, McNaughton BL (1994) Reactivation of hippocampal ensemble memories during sleep. Science 265:676-679.

Wouterlood FG, Saldana E, Witter MP (1990) Projection from the nucleus reuniens thalami to the hippocampal region: light and electron microscopic tracing study in the rat with the anterograde tracer Phaseolus vulgaris-leucoagglutinin. J Comp Neurol 296:179-203. 